\title{
FULLY DEVELOPED TURBULENCE VIA FEIGENBAUM'S PERIOD-DOUBLING BIFURCATIONS
}

\author{
Minh Duong-van
}

Presented at the International Conference on the Physics of Chaos and Systems Far from Equilibrium held in Monterey, California, January 11-14, 1987

(To be published in the above proceedings)

\section{August 1987}

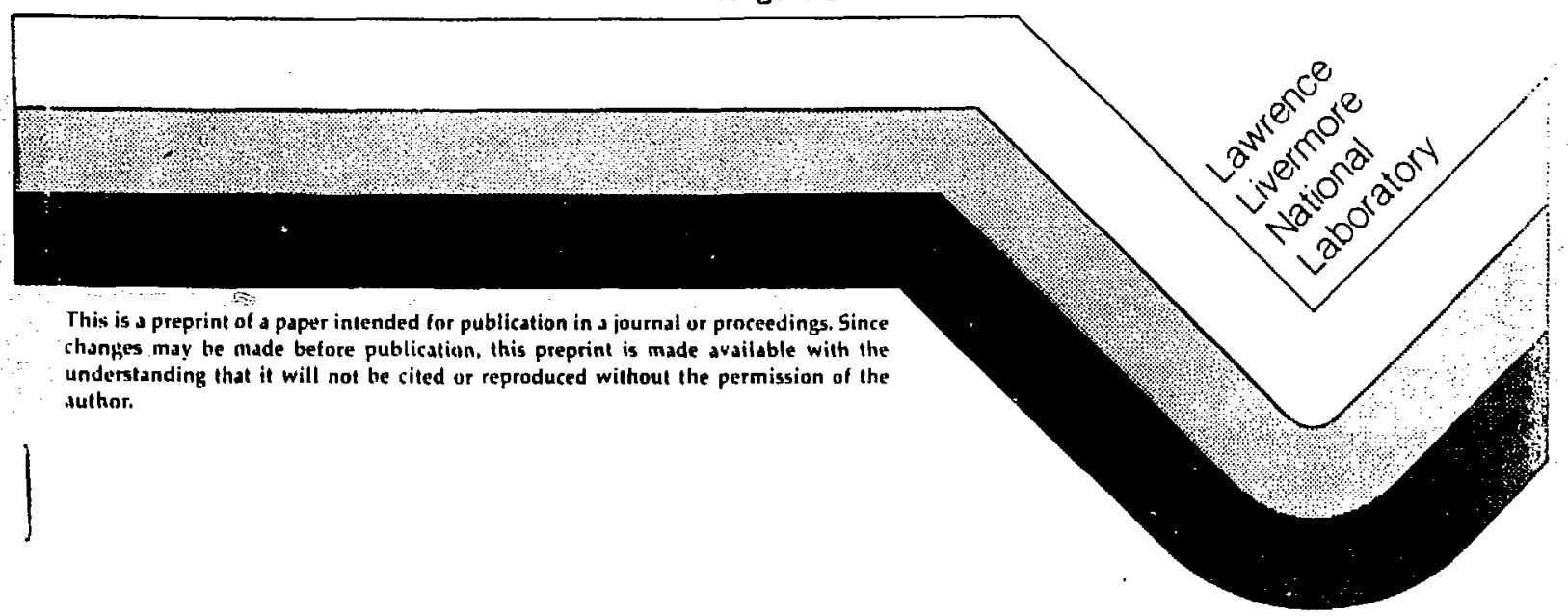




\title{
FULLY DEVELOPED TURBULENCE VIA FEIGENBAUM'S \\ PERIOD-DOUBLING BIFURCATIONS
}

\author{
Minh Duong-van \\ Physics Department \\ Lawrence Livermore National Laboratory \\ University of California, Livermore, CA 94550
}

\begin{abstract}
Since its publication in 1978, Feigenbaum's predictions of the onset of turbulence via period-doubling bifurcations have been thoroughly borne out experimentally. In this paper, Feigenbaum's theory is extended into the regime in which we expect to see fully developed turbulence. We develop a method of averaging that imposes correlations in the fluctuating system generated by this map. With this averaging method, the field variable is obtained by coarse-graining. while microscopic fluctuations are preserved in all averaging scales. Fully developed turbulence will be shown to be a result of microscopic fluctuations with proper averaging. Furthermore, this model preserves Feigenbaum's results on the physics of bifurcations at the onset of turbulence while yielding additional physics both at the onset of turbulence and in the fully developed turbulence regime: with this method of averaging,
\end{abstract}

1) the observed smaller ratio of power spectra of successive bifurcations than that predicted by quadratic-maximum maps;

2) the manifestation of a Kolmogorov spectrum, with a slope slightly flatter than -2 beyond the onset of turbulence;

3) intermittency in the time series in the regime where it is not observed by these maps without averaging; and

4) a fractal dimension of about 6-10 in the fully developed turbulence regime. 
A number of phenomena in nature, as a universal characteristic, have complex microscopic behavior that underlies macroscopic manifestations. One is the critical phenomenon, another is fully developed turbulence. In these problems, microscopic fluctuations average out when larger scales are considered and the continuum limit is achieved by averaging over larger and larger scales.

The simplest example one can use to study this passage to the continuum limit is hydrodynamics cast in a microscopic framework. The standard method of averaging atomic or molecular fluctuations yields the classical hydrodynamic equation. But a new method of averaging is required if we are to treat properly the case in which the fluctuations persist out to the macroscopic scale, the scale on which macroscopic measurements are made.

In critical phenomena, a renormalization-group technique was developed to handle the physics of many scales. 1 In turbulence, because of the lack of formal theories, we propose to approach this problem phenomenologically. We assume that fully developed turbulence is a macroscopic manifestation of microscopic chaos (fluctuations). We begin by assuming some deterministic model of fluctuations whose details are unimportant provided that it has the following properties:

1) The representation of the fluctuations can be extracted from the field equations of the dymamical systems. This representation as function of time is completely deterministic. For example, the fluctuations can be represented by a time evolution recursion relation.

2) The representation is sufficiently nonlinear to guarantee significant coupling between all wave components so that solutions cannot be linearly superposed.

3) The dymamical equations manifest many field modes that are excited, and even in the mean field limit, in each region however small, the fleld fluctuates significantly with a kind of chaotic motion that leads to a high degree of chaos in the fluctuations. 
4) On the smallest time scale or in the smallest :egion the fluctuations are large. At larger scales, the effect of the fluctuations become smaller and smaller until we reach a scale at which they become negligible. However, there is no physical limit in which these fluctuations vanish completely.

In this paper, we build a model that satisfies the above criteria. Microscopic fluctuations are proposed to be governed by a quadratic-maximum map (we use the logistic map, which satisfied properties 1 and 2). We develop a method of averaging that imposes correlations in the fluctuating system generated by this map. With this averaging method, the field variable is obtained by coarse-graining, while microscopic fluctuations are preserved in all averaging scales. Fully developed turbulence will be shown to be a result of microscopic fluctuations with proper averaging. Furthermore, this model preserves Feigenbaum's results on the physics of bifurcations and the onset of turbulence while yielding additional physics both at the onset of turbulence and in the fully developed turbulence regime: with this method of averaging,

1) the observed smaller ratio of power spectra of successive bifurcations than that predicted by quadratic-maximum maps;

2) the manifestation of a Kolmogorov spectmum, with a slope slightly flatter than -2 beyond the onset of turbulence;

3) intermittency in the time series in the regime where it is not observed by these maps without averaging; and

4) a fractal dimension of about 6-10 in the fully developed turbulence regime.

Recent discoveries of Feigenbaum ${ }^{2}$ on the onset of turbulence provide a firm foundation upon which we build our model of fully developed turbulence. Feigenbaum approaches turbulence not from the classical point of view, but rather starts by observing that given initial conditions that are infinitesimally different, 
wildly different outcomes are possible, that almost any nonlinear system exhibits chaotic behavior. The starting point is the assumption that a deterministic quadratic-maximum map has enough nonlinearity that it describes microscopic fluctuations. One such map is the logistic map,

$$
f \equiv u_{n+1}=\lambda u_{n}\left(1-u_{n}\right)
$$

where $u$ denotes the fluctuating quantity controlled by a stress parameter $\lambda$. When $\lambda$ is suitably varied, the system described by $u$ changes from simple to chaotic. More precisely, for some range of the parameter value, the system exhibits an orderly periodic behavior; that is, the system's behavlor reproduces itself every period of time $T$. When $\lambda$ is increased further, the systems requires two intervals of $T$ to repeat itself: the period of the system has doubled. As $\lambda$ is increased still further, the period continues to successively double up to infinity. Denoting by $\lambda_{\mathrm{m}}$ the value of the parameter at which the period of the system doubles for the $\mathrm{m}^{\text {th }}$ time, Feigenbaum found that $\lambda_{m}$ converges to $\lambda_{\infty}$ for large $\mathrm{m}$ :

$$
\lambda_{\infty}-\lambda_{m} \propto \delta^{-m}
$$

with $\delta=4.6692 \ldots .$. a universal vaifa. This universality has been repeatedly confirmed experimentally. $3,4,5$

In spite of the success of this theory, the problem of fully developed turbulence has not been completely understood from this picture. The simplest unanswered question raised by Feigenbaum's work is why the Fourier spectrum is chaotic and flat in frequency when the stress parameter $\lambda$ reaches its maximally chaotic value of $\lambda=4$. Experimentally the Kolmogorov spectrum is slightly flatter than -2 for this type of turbulence. 6,7 
Another question is the discrepancy between the predicted and measured ratio $R$ of the power spectra of successive harmonics at the onset of turbulence, $\lambda=\lambda_{\infty}$. The theory ${ }^{2}$ predicts $R \approx 43$ while several experimental data ${ }^{8}$ yield much smaller $R$ $=7$ to 12 .

To expand the theory developed by Feigenbaurn into the fully developed turbulence regime, we develop a new method of averaging the fluctuations generated by the quadratic-maximum map. We cast the logistic map a microscopic map which describes the fluctuations of turbulent systems in a microscopic scale. We impose an averaging scheme onto these microscopic fluctuations to bring the system into macroscopic domains. This method of averaging is an analog of of the coarse-graining technique in critical phenomena. ${ }^{1}$ Coarse-graining is a method of imposing macroscopic correlations into microscopic fluctuations. It transforms a system from a discrete state to a near-continuum one. In the Ising model, for example, discrete spins become essentially continuous after coarse-graining as the first step of a Kadanoff transformation. Suppose we have block spins each of which can assume two values, +1 or -1 . We combine 8 blocks to form a new block. The new block spin, i.e., the mean of 8 old spins, can assume one of the nine values $( \pm 1$, $\pm \frac{6}{8}, \pm \frac{4}{8}, 0$ ). Coarse-graining degrades (but does not completely destroys) the discreteness of the system. In the following sections, we will discuss the details of our model.

\section{A NEW METHOD OF AVERAGING MICROSCOPIC FLUCTUATIONS:}

Our method of coarse-graining was recently introduced by $\mathbf{M}$. Pound and the author ${ }^{9}$ to average out nicroscopic fluctuations in a lattice-gas automation in order to obtain the macroscopic velocity fields. This was done in spatial configuration, as in the Ising model. For time series, we employ the same technique as an one-dimensional analog of the cellular automata coarse-graining. 
Suppose nature's fluctuations are sufficiently modeled by the microscopic fluctuations $u$ of the logistic map $f \equiv u_{n+1}=\lambda u_{n}\left(1-u_{n}\right)$. The first $N$ terms of the time series are $u_{1}, u_{2}, \ldots ., \mathrm{N}$. Call $v_{1}$ the average value of consecutive $u$ 's of time length $\zeta=m \Delta t$, where $\Delta t$ is the time lapse between consecutive microscopic fluctuations, conveniently set to be $\Delta t=1$ :

$$
v_{1}=\frac{1}{M} \sum_{n=1}^{M} u_{n}=\frac{1}{M} \sum_{m=1}^{M} f_{\left(u_{0}\right)}^{m}
$$

where $f^{m}\left(u_{0}\right)$ is the value of $f$ after $m$ operations on the initial value $u_{0}$. In our notation, $\dot{j}_{\text {may. }}=\mathrm{N}-\mathrm{M}+1$.

The next averaged value $v_{2}$ is then obtained by repeating the averaging process with a shift of one unit of $n$. The physical reason for this choice of averaging will be discussed in the subsequent section.

$$
v_{2}=\frac{1}{M} \sum_{n=2}^{M+1} u_{n}=\frac{1}{M} \sum_{m=2}^{M+1} f^{m}\left(u_{0}\right)
$$

The last term is:

$$
v_{N-M+1}=\frac{1}{M} \sum_{n=N-M+1}^{N} u_{n}=\frac{1}{m} \sum_{m=N-M+1}^{N} f m_{\left(u_{0}\right)}
$$

The new time series of the averaged fluctuations can be obtained by successive averaging. In the case of cellular atomata, u can represent, for example, the microscopic velocity of each point on the lattice. If we carry out a spatial average along the lines first described, we obtain the observed velocity field, as mentioned earlier. 9 
in this model, we start with a deterministic equation (the logistic map, for example) where all iterations are deterministic. The values of all the velocities v's are also deterministic, with explicitly known functional forms written in terms of the initial condition $u_{0^{*}}$

This special method of averaging deserves some explanation. The most common method of averaging. performed by other workers in cellular automata and related fields, uses the maximum shift of $\Delta n=M+1$, rather than $\Delta n=1$. This means, for example, in the case of Fig, $1, v_{1}=\frac{1}{4}\left(u_{1}+u_{2}+u_{3}+u_{4}\right)$ and $v_{2}=\frac{1}{4}\left(u_{5}+\dot{u}_{6}+\right.$ $\left.u_{7}+u_{8}\right)$, rather than $v_{2}=\frac{1}{4}\left(u_{2}+u_{3}+u_{4}+u_{5}\right)$ as in our method. For the shift $\Delta n=$ $M+1$, if the Fourier transform of the $u$ 's is flat after averaging, the Fourier transform of the v's remains flat. However, in our method, when the shift of $\Delta \mathrm{n}=1$, averaging the Fourier transform of the averaged v's approaches a slope slightly flatter than - 2. This was observed in our cellular automaton. 9 In our method, the length $\zeta=\mathrm{M} \Delta \mathrm{t}$ is actually the imposed correlation onto the microscopic fluctuation system. The shift of $\Delta \mathrm{n}=\Delta \mathrm{t}=1$ guarantee the preservation of microscopic fluctuations while the mean field $\mathrm{v}$ is obtained by averaging u's. For large $\zeta=M$ (that is, $\Delta t \equiv 1$ ). the effect of this microscopic fluctuation becomes small; as $\zeta$ is larger, this effect becomes smaller. But there is no limit in this discrete case in which the microscopic fluctuation vanishes completely. Figure 2 illustrates this type of physics. The chaotic fluctuation is generated from the logistic map. with $\lambda=$ 4 (Fig. 1a). Figure 1b shows the averaged $V$ 's for $M=50$. The "trends" seen in these figures reflects the correlation imposed by this method of averaging. The microscopic fluctuations relative to the mean field decrease with increasing $M$. The physics of this averaging method is now investigated. 


\section{RESULTS OF AVERAGING RANDOM FLUCTUATIONS.}

This work on randorn fluctuations is recently introduced by $M$. D. Feit and the author. ${ }^{10}$ Consider a random time series $f_{j}=f\left(t_{j}\right), j=1$ to $N$, with discrete Fourier träsiform $g(\omega)$ :

$$
g(\omega)=\sum_{j} f_{j} \exp \left(i \omega t_{j}\right)
$$

Here $|g(\omega)|^{2}$ is the power spectmum; its ensemble average is independent of frequency $\omega$ for a white noise source. We attempt to measure this process on the macroscopic level with a physical device of limited temporal resolution. Suppose the impulse response of the device is $W(t)$, then the measured signal will be $F(t)=$ $f(t) * W(t)$ with power spectrum $|F(\omega)|^{2}=|g(\omega)|^{2}|V(\omega)|^{2}$. Here, the star denotes convolution and $V(\omega)$ is the Fourier transform of the impulse response $W(t)$.

The simfiest possible case is that of a running average over a time interval $M \Delta t$ ie: $W(t)=1$ for $0<t<M \Delta t$ and 0 otherwise. In this case, the measured power spectrum is proportional to

$$
|V(\omega)|^{2}=(\sin \omega T / 2 / \sin \omega \Delta t / 2)^{2}
$$

This function is plotted in Fig. $2 a$ for $N=2048$ and a window of $M=250 \Delta t$. Fig. $2 b$ is for $N=500$. It is seen that the spectrum displays an app ent power law behavior in the high frequency range with exponent slightly flatter than -2 . The apparent leveling at $i: 3$ highest $\omega$ is due. to the finite spectral width and periodicity of the finite Fourier transform. The approximate slope of -1.9 is nearly independent of the size of the window as long as the window $M$ is small relative to $N$. Physically, we can think of $\mathrm{M} \Delta \mathrm{t}$ as a correlation time. Then statistically relevant results require $\mathrm{M}$ 
$\ll N \Delta t$. The continuum limit should be approached when $\Delta t \ll k \Delta t \ll N \Delta t$. For a smaller $M$, the effect of discreteness will be manifested.

This result can be understood by taking the logarithmic derivative $\operatorname{d\ell n}|V(\omega)|^{2} / d \ln \omega$.

$$
a \ln |V(\omega)|^{2} d \ell n \omega=\frac{\omega M \sin \omega M}{1-\cos \omega M}-\omega \Delta t \cot \left(\frac{\omega \Delta t}{2}\right)
$$

The slowiy varying envelope seen in Fig. 1 is due to the denominator of Eq. 6 . The resulting slope varies from -2 to about -1.56 as $\omega \Delta t$ varies over 0 and $\pi$; the average value is $-1,86$. This result is very siggestive in iight of experimentally determined power spectra of turbulent systems on the one hand, and fluctuations near a critical point on the other (the critical exponent for the correlation function is $-2+\eta$ where $\eta \approx .14$ experimentally).

Assuming isotropic turbulence, Eq. 7 can be used to calculate the correlation spectrum $G(k)$ parameterized conventionally as:

$$
G(k) \sim k^{-2+\eta}=k^{s}
$$

For infinite system, $\eta=0$ (as in mean-field theory of continuum system). For discrete-finite system, $\eta$ can be calculated from Eq. 7.

$$
\eta=\frac{2 \ln \left(\frac{M}{2} \sin \frac{\pi}{M}\right)}{\ln \frac{M}{2}}
$$

This is exactly the logarithmic correction. For $M=50$ (see Section 2 ), $\eta=.14$ and the "Kolmogorov" slope is -1.86 . 
As a demonstration of these ideas, we constructed a finite stochastic time series of $N=2048$ values with a random number generator. The power spectrum of the series was found to be flat as expected. The "measured" time series was generated by using a ruming average of width $200 \Delta \mathrm{t}$. The trend can be made more evident by performing a running average in frequency as well; this has been done in Fig. 2 over a frequency width of $20 \Delta \omega$. The slope of the spectrum is consistent with -1.9 approximately.

We conclude that the slope of $-2+\eta$ where $\eta$ is a small positive correction is characteristic of a discrete process with limited time correlation, in distinction to the slope of -2 characteristic of a continuum. Furthermore, the power law spectrum is a natural consequence of finite spatial or temporal resolution measurernents of finite time series. The imposition of correlation through finite resolution observation is enough to lead to power law spectra.

\section{RESULTS OF AVERAGING CHAOTIC FLUCTUATIONS.}

We now maike use of this method of averaging to study the physics of chaotic fluctuations.

We apply this coarse-graining to time series generated by the logistic map (Eq. 1). Figure 3 shows the Lyaponov exponent $\sigma$ for various values of $\lambda$ in (1):

$$
\sigma=\lim _{N \rightarrow \infty} \frac{1}{N} \sum_{i} \ln \mid \frac{d f}{d u_{i}}-1
$$

We will study the physics produced by marching through the exponent $\sigma$. 
4. MODIFICATION OF RATIO OF SUCCESSIVE BIFURCATION POWER SPECTRA AT $\underline{\lambda}_{\infty}$

Period doublings occur in the interval $3<\lambda \leq 3.56980$. Between $\lambda_{\infty}=$ 3.56980 and $\lambda=4$, there are several windows of $\sigma<0$ where periodicities of different periods are observed. Figures $4 a$ to $4 \mathrm{e}$ are the power spectra of the logistic map at $\lambda_{\infty}$ for the running time average of width $M=1$ to 300 respectively, $(\Delta t \equiv 1)$. The ratio $R$ of the power of the main frequency $F$ and its first harmonic $F / 2, R=P(F) / P(F / 2)$ ) versus the running time average $M$ is shown in Fig. 5 . The value of $R$ is invariant as long as $\tau=2^{n \Delta t}$ for $n=1, N$ as expected because of the bifurcation nature in this range of $\lambda$. The interesting result is scen when $M \neq 2^{n}$. The ratio $R$ can be shown to decrease with $M$ as

$$
R \times M^{-2}
$$

Thus, the ratio of successive harmonics decreases as the time response (window) of the measuring probe increases. To be consistent with Libchaber's data, ${ }^{10}$ we use $M$ $\simeq 50$. Historically, the theoretical prediction ${ }^{2}$ of Feigenbaum $R_{a}=43(8.2 \mathrm{db})$ is the amplitude ratio and the experimental data ${ }^{8}$ of $R=8.2 \mathrm{db}$ is the power ratio (square of the amplitude). This is therefore not an agreement between predictions and the data, as commonly believed. Only after we averaged out the data generated by the logistic map was this discrepancy remedied. We found that for $M=50$ the theory and experimental data agree.

\section{MODIFICATION OF THE SLOPE OF THE POWER SPECTRA AT $\lambda=4$.}

At $\lambda=4$, if we want to claim that fully developed turbulence is a consequence of period doubling bifurcations, we should expect the power spectrum to mimic the 
approyimate -1.9 power. Figure $6 \mathrm{a}$ shows the power spectrum of the u's generated by Eq. 1 with $\lambda=4$. Without coarse-graining, it is flat as expected fron the stochastic nature of the logistic map.

We will see now how this new method of averaging change the nature of the outcome of the power spectra of the logistic map at $\lambda=4$. Figures $6 \mathrm{~b}$ to $6 \mathrm{~d}$ show the power spectra with a time averaging window $M=2$ to 20 . We can see that the high frequency is decreased as a consequence of this time average.

When $M=100$, the power spectrum is almost saturated to a power law with the slope approximately -1.9 in the high and medium frequencies range (Fig. 6e). Increasing values of $M$ to 300 the power spectrum is still approximately saturated as a power law of -1.9 (Figs. 6f). This result coincides with various experimental data on fully developed turbulence in the inertial range.

We now want to stress a basic point: there is perhaps no continuum limit for turbulence. By coarse-graining the microscopic system with larger $M$, the macroscopic variables still retain fluctuations. The fluctuations do not vanish in any limit of $M$. The averaging process is irreversible: Once the averaging is done, the Iuctuations are unrecoverable. We believe that any attempt to understand fully developed turbulence from hydrodynamic equations where fluctuations are ignored will suffer the same fate as Landau's theory of critical phenomena.

In experiments, the frequencies are averaged out according to the resolution of the frequency analyzer. To simulate this effect, we average the frequencies with a width of 100 bins and Fig.7 shows the same result as Fig. 63 with $\Delta \omega=100$. The power law spectrum is consistent with fully developed turbulence data. 


\section{INTERMITTENCY IN THE TIME AVERAGED LOGISTIC MAP.}

In real fluids, between laminar and turbulent regimes, there is a phenomenon called intermittency. ${ }^{8}$ We use the logistic map at this regime to study the intermittency phenomenon, and we choose $\lambda=3.62$ (see Fig. 3). The time series is represented in Fig. 8a. No intermittency is indicated. This is also shown in a magnified version, Fig. 8b. However, when the series is time averaged with a window $M=67$ definite intermittency is observed. This shows a definite long time (large scale) correlation of the map (Fig, 9). The same is true for any other arbitrary value of the time window $M$, say, $M=299$. (Fig. 10).

In another regime near the fully developed turbulence, $\lambda=3.857$, we studied the behavior of this type of intermittency. Figures $11 \mathrm{a}$ to $11 \mathrm{e}$ show the intermittency phenomenon for various values of $M$. Thus, this phenomenon is global and robust, i.e., independent of $\lambda$ and $m$ for $\lambda>0$.

\section{INCREASE OF FRACTAL DIMENSION BY AVERAGING}

Data of fully developed turbulence shows fractal dimensions of 6-10, much larger than $D_{F}=1$ as calculated from the logistic map at $\lambda=4$.

To calculate ${ }^{11} \mathrm{D}_{\mathrm{F}}$, we generate a time series of the logistic map at $\lambda=4$, to get $X n\left(n=1\right.$. N). We build a $D_{i m b}$ dimensional space vectors $V_{n}$ with: 


$$
v_{1}=\left(\begin{array}{l}
x_{1} \\
x_{2} \\
x_{3} \\
\cdot \\
\cdot \\
\cdot \\
x_{D_{\mathrm{imb}}}
\end{array}\right)
$$

$$
v_{2}=\left(\begin{array}{l}
x_{2} \\
x_{3} \\
x_{4} \\
\cdot \\
\cdot \\
\cdot \\
x_{\mathrm{pmb}_{\mathrm{mb}}}+1
\end{array}\right)
$$

In this new space, we calculate the Euclidean norms ln's. For example, when $D_{\mathrm{imb}}=2:$

$$
\begin{aligned}
& \ell_{1}=\sqrt{\left(x_{1}-x_{2}\right)^{2}+\left(x_{2}-x_{3}\right)^{2}} \\
& \ell_{2}=\sqrt{\left(x_{2}-x_{3}\right)^{2}+\left(x_{3}-x_{4}\right)^{2}}
\end{aligned}
$$

For a given sphere of radius $R$, we count the number of $\ell$ 's that are less than $R$. We postulated that this number $L(R)$ obeys:

$$
L(R)-R^{p}
$$


For the logistic map at $\lambda=4$, uring this method and for all $\mathrm{D}_{\mathrm{imb}}$ between 2 and 50 , we found $D_{F} \approx 1.0$ (Fig. 12). When this series is time averaged (say $M=50$ ) the fractal dimension saturates with the imbedding dimensions. We found with $M=50$, $D_{F} \sim 8 \pm 2$, consistent with experimental data.

In conclusion, we have developed a method of averaging that can be used to understand data of fully developed turbulence.

At the onset of turbulence, Feigenbaum has shown that turbulent systems manifest period doubling bifurcations with universal parameters $\delta=4.669$ and $\alpha=$ 2.503. This prediction, which describes the modern approach to turbulence, has been observed in various experiments.

However, the theoretical prediction of the ratio $R$ of the power spectra of successive bifurcations is much higher than the measured data. Furthermore, if one attempts to extend this theory to the fully developed turbulence regime one gets a fractal dimension $D_{F}=1$, the slope of the power spectrum $S=0$, and one predicts no global intermittency in the regime between the onset and fully developed turbulence. Experimentally, $D_{F} \simeq 6$ to $10, S \simeq-5.3$ and the global intermittency is observed in various systerns.

Influenced by our calculations with the Lattice Gas Cellular Automaton and by renormalization group methods, we developed a method of 'averaging' the nonlinear fluctuations generated by the quadratic maximum map used by Feigenbaum. With this averaging scheme we achieve three important results. First, we recover the universal; values of $\delta$ and $\alpha$. Secondly, the value of $R$ is now consistent with Liuchaber's experimental data. Finally, we obtain the observed fractal dimension $\left(D_{F}=6-10\right)$ and a calculated power spectrum with a slope $S$ approximately equal to $-5 / 3$, consistent with Kolmogorov and with experiments. The global intermittency is also recovered. This new work provides a theory that bridges the classical and the 
modern treatments of turbillence. Our predictions are consistent with existing data at the onset of turbulence and in the fully developed turbulence regimes.

What we have discovered here is that, in the microscopic scale, nature manifests a brownian motion turbulence. But when a measurement is made onto the microscopic scale to bring the system into the macroscopic scale, the physics will change drastically in terms of intermittency, steep Kolmogorov slope and large fractal dimension.

\section{ACKOWLEDGMENT}

1 thank J. Nuckolls and B. Tarter for encouraging this research. Various collaborations with $M$. Feit, M. Pound, P. Keller are appreciated. I benefited greatly from conversations with M. Feigenbaum, K. Wilson, R. Minich, G. Hedstrom, J. Wong, D. Levermore in the course of the research. Lastly, I wish to thank Joni Schuld and Han Wen for the finalization of the manuscript.

Work performed under the auspices of the U.S. Department of Energy by the Lawrence Livermore National Laboratory under contract number W-7405-ENG-48.

\section{DISCLAIMER}

This report was prepared as an account of work sponsored by an agency of the United States Government. Neither the United States Government nor any agency thereof, nor any of their employees, makes any watranty, express or implied, or assumes any legal liability or responsibility for the accuracy, completeness, or usefulness of any information, apparatus, product, or process disclosed, or tepresents that its use would not infringe privately owned rights. Reference herein to any specific commercial product, process, or service by trade name, trademark. manufacturer, or otherwise does not necessarily constitute or imply its endorsement, recommendation, or favoring by the United States Government or any agency thereof. The vicws and opinions of authors expressed herein do not necessarily state or reflect those of the United States Government of any agency thereof. 


\section{REFERENCES}

1. K. Wilson, Rev. Mod. Phys. 47,773 (1975).

2. M.J. Feigenbaum, J. Stat. Phys. 19, 25 (1978); 21, 669 (1979); Phys. Lett. 74A, 375 (1979); Common. Math. Phys. 77.65 (1980).

3. A. Libchaber and J. Maurer, J. Phys. (Paris) 41, C3-51 (1980); J. Physique Lett. 40 (1979) L-419.

4. J.P. Gollup, S.V. Benson, and J. Steinman, Ann. N.Y. Acad, Sci. 357, 22 (1980).

5. For an excellent review on this subject, see P. Cvitanovic, Universality in Chaos, Adam Hilger Ltd. Publisher.

6. L.S. Huang, 1985 Ph.D. Thesis, University of Southern California, Los Angeles, CA 90089.

7. F.K. Browand, Physica 18D (1986) 135-148.

8. A. Libchaber and J. Mauer, Nonlinear Phenomena at Phase Transitions and Instabilities (ed. T. Riste) 259-86 (1982).

9. M. Duong-van, M. Pound, M. Feit and P. Keller, Physica 23D (1986) 448-454.

10. M. Duong-van and M. Feit, Phys. Lett. A, 119 (1987) 388-390.

11. M. Duong-van "Instabilities and Turbulence and the Physics of Fixed Points," p. 171-178, Dîmensions and Entropies in Chaotic Systems, Springer-Verlag, 1986 (G. Mayer-Kress, Ed.) 


\section{FIGURE CAPTIONS}

Figure 1a: Time series of the logistic map for $\lambda=4, M=1$

Figure 1b; Time series of the logistic map for $\lambda=4, M=50$

Figure 2a: $\quad$ The power spectrum of Equation (6) with $\mathrm{M}=250$.

Figure 2b: The power spectrum of Equation (6) with $M=500$. The dashed line is for $P(\omega) \sim \omega^{-5 / 3}$.

Figure 3: $\quad$ The Lyaponov exponent versus $\lambda$ of the logistic map.

Figure 4a: The power spectrum of the logistic map at $\lambda=3.56980$ with $M=1$.

Figure 4b: The power spectrum of the logistic map at $\lambda=3.56980$ with $M=5$.

Figure 4c: The power spectrum of the logistic map at $\lambda=3.56980$ with $\mathrm{M}=8$.

Figure 4d: The power spectrum of the logistic map at $\lambda=3.56980$ with $\mathrm{M}=128$.

Figure 4e: The power spectrum of the logistic map at $\lambda=3.56980$ with $M=300$.

Figure 5: $\quad$ The ratio $R$ of two successive peaks of the power spectrums as a function of the averaging window $M$.

Figure 6a: The power spectrum of the logistic map at $\lambda=4.0$ and $M=1$.

Figure 6b: The power spectrum of the logistic map at $\lambda=4.0$ and $M=2$.

Figure 6c: The power spectrum of the logistic map at $\lambda=4.0$ and $M=5$.

Figure 6d: The power spectrum of the logistic map at $\lambda=4.0$ and $M=20$.

Figure 6e: The power spectrum of the logistic map at $\lambda=4.0$ and $M=100$.

Figure 6f: The power spectrum of the logistic map at $\lambda=4.0$ and $M=300$.

Figure 7: The power spectrum of the logistic map at $\lambda=4.0, M=300$ and the frequency averaging of 100.

Figure 8a: The time series of the logistic map at $\lambda=3.62$ and $M=1$.

Figure 8b: The time series of the logistic map at $\lambda=3.62$ and $M=1$ (expanded horizontal scale).

Figure 9: The time series of the logistic map at $\lambda=3.62$ and $M=67$. 
Figure 10: The time series of the !ogistic map at $\lambda=3.62$ and $M=299$.

Figure 11a: The time series of the logistic map at $\lambda=3.875$ and $M=5\left(X_{0}=\right.$ .0438).

Figure 11b: The time series of the logistic map at $\lambda=3.875$ and $M=6\left(X_{0}=\right.$ .0438).

Figure 11c: The time series of the logistic map at $\lambda=3.875$ and $M=4\left(X_{0}=\right.$ .0438).

Figure 11d: The time series of the logistic map at $\lambda=3.875$ and $M=10\left(X_{0}=\right.$ $.0438)$.

Figure 11e: The time series of the logistic map at $\lambda=3.875$ and $M=50\left(X_{0}=\right.$ .0438).

Figure 12: The fractal dimension and $L(R)$ vs. $R$ plot for the logistic map at $\lambda=$ 4.0.

Figure 13: The plot of $L(R)$ vs. $R$ for the logistic map at $\lambda=4.0$ and $M=50$. 

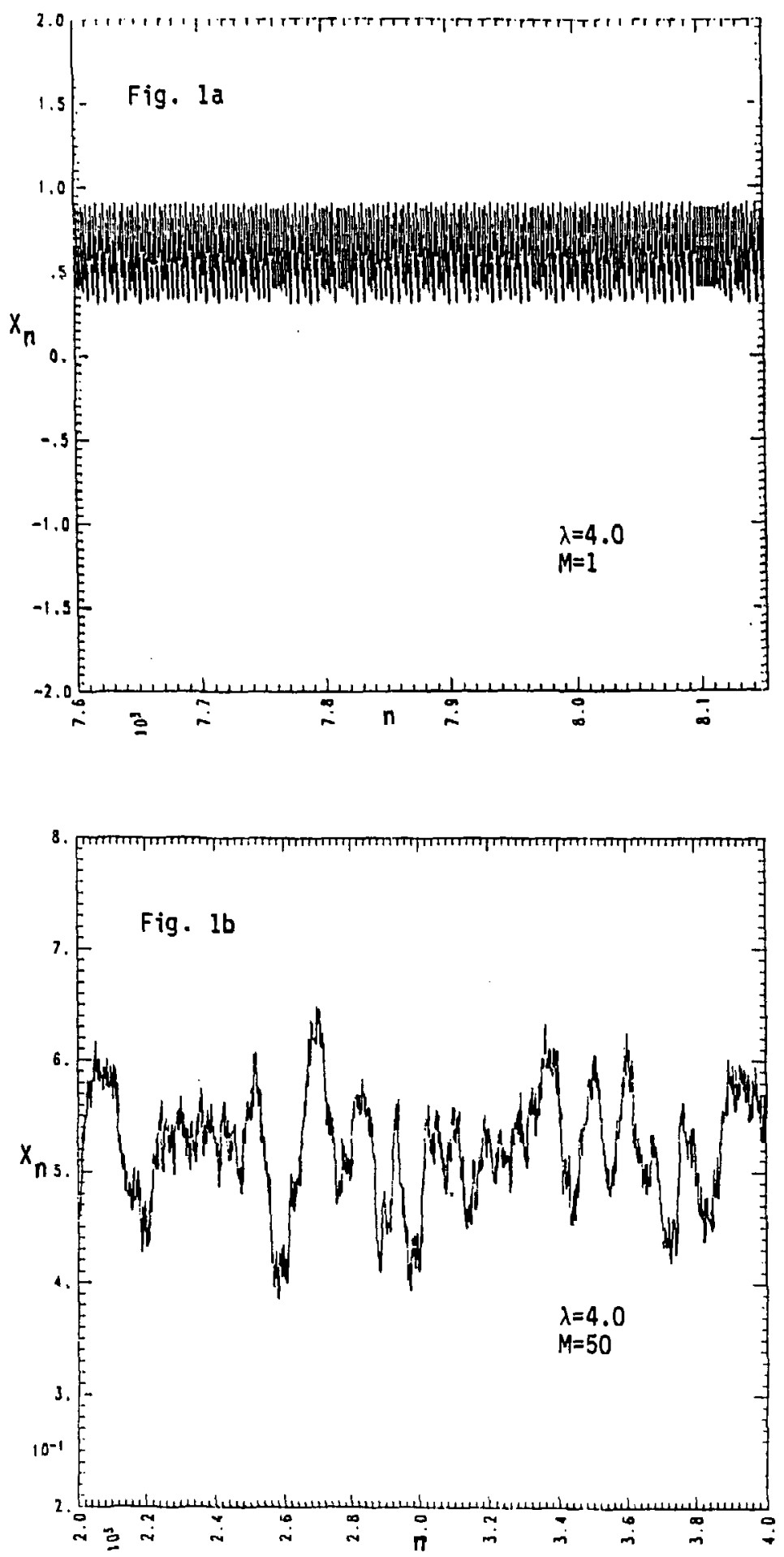

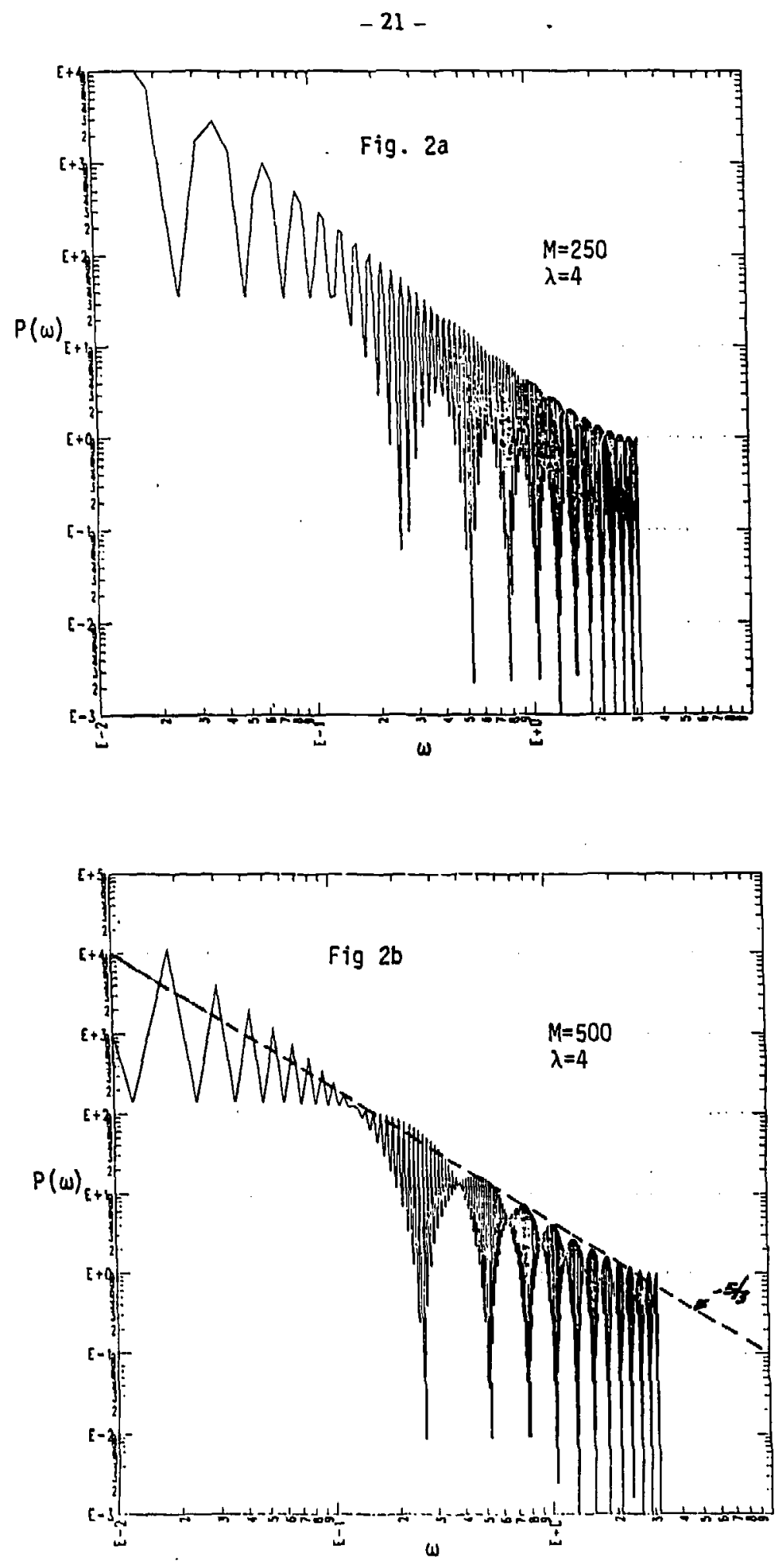
Е

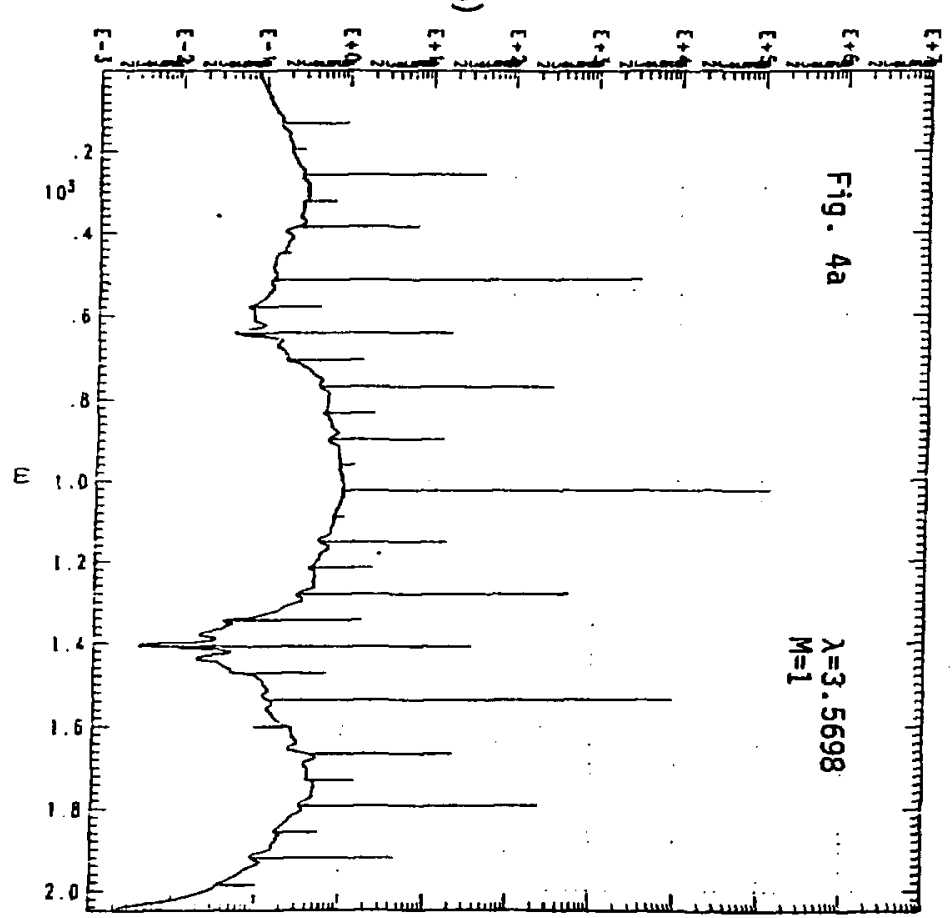

The Lyaponov Exponent

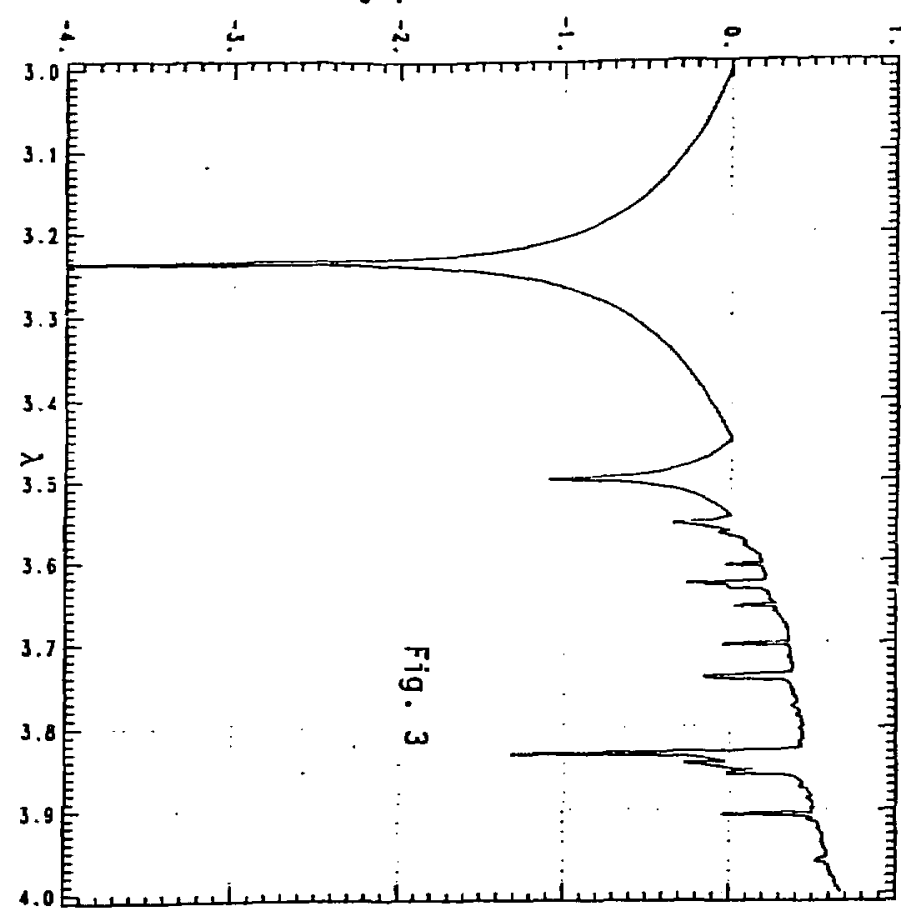



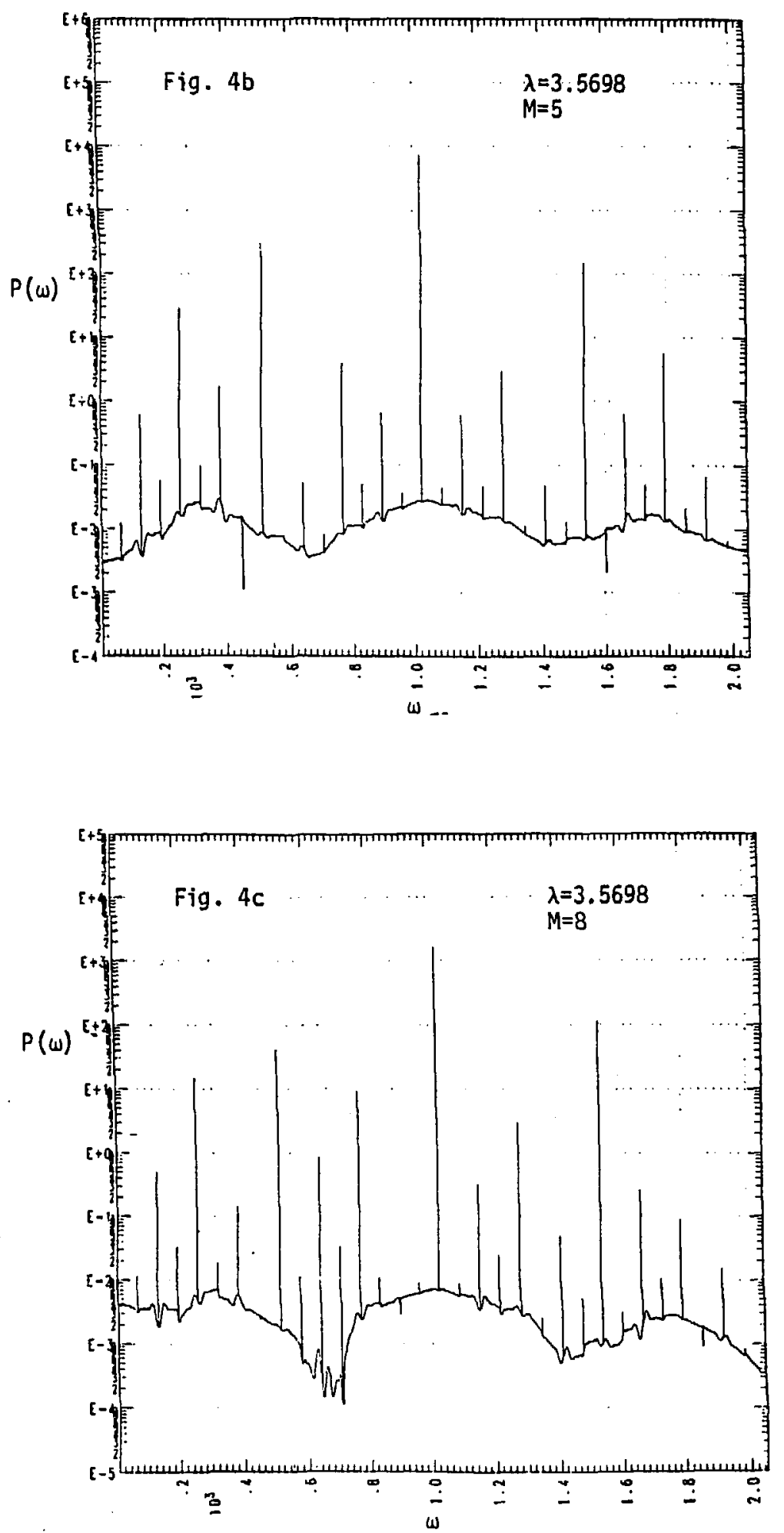

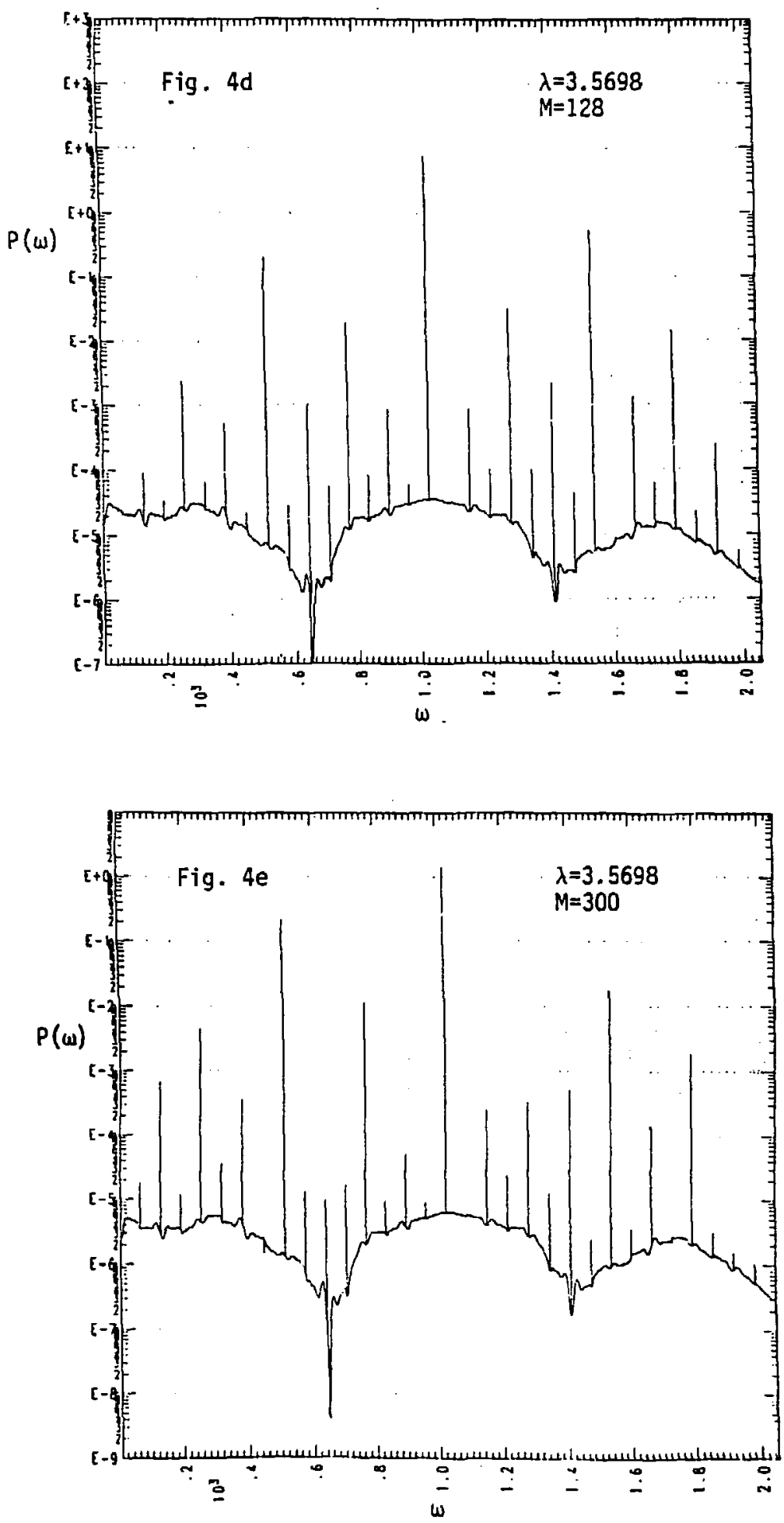

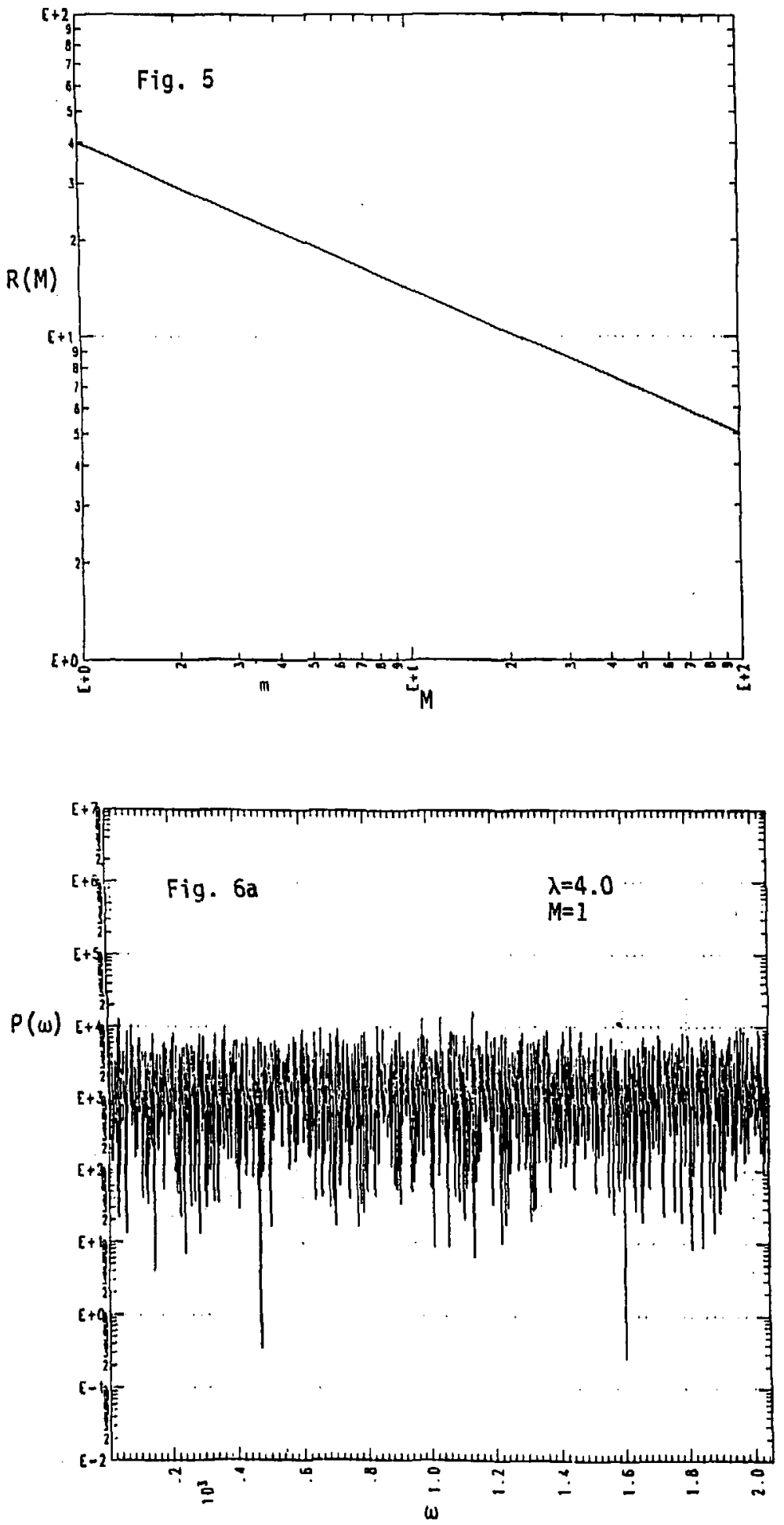

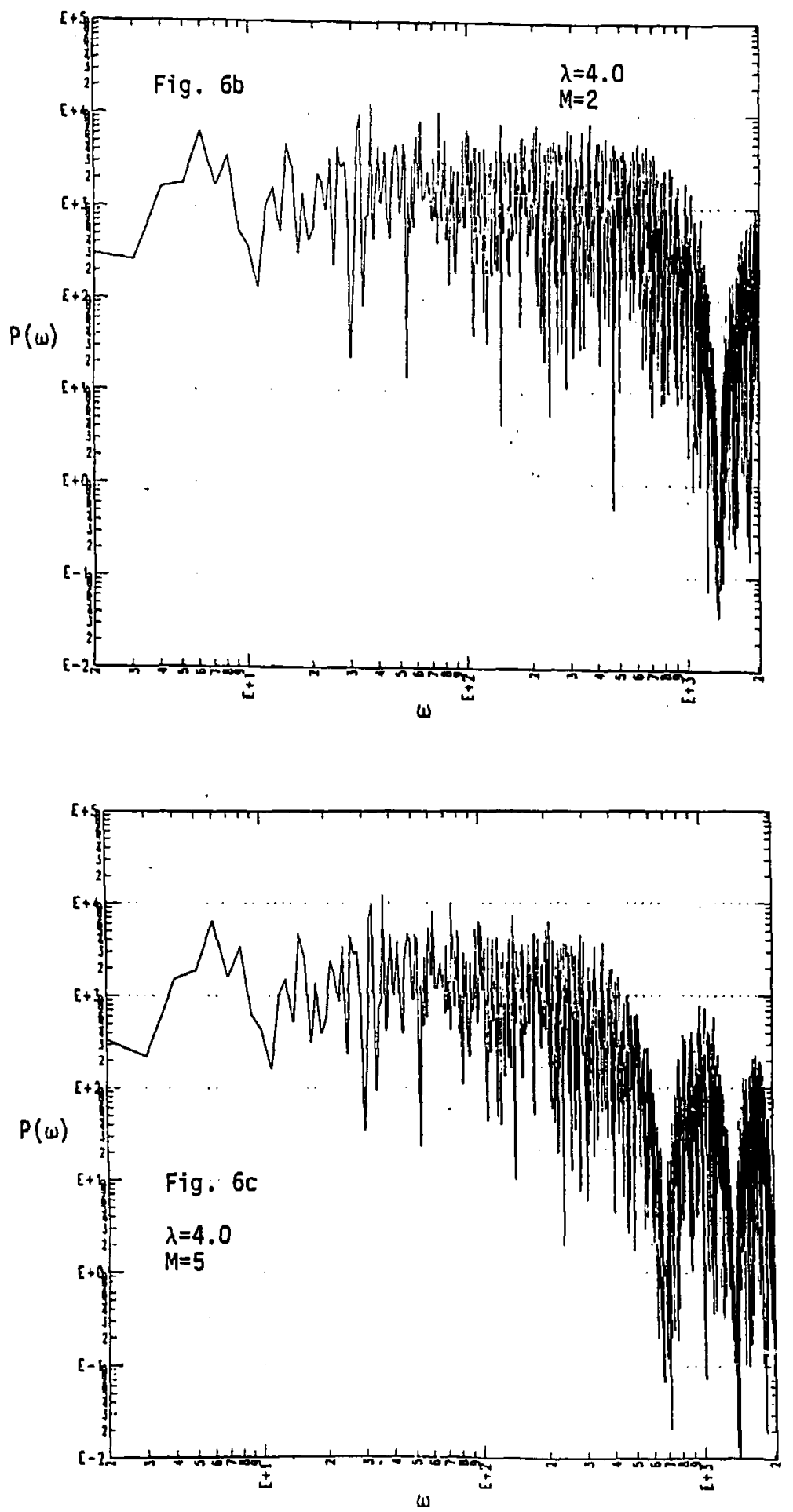
-27 -
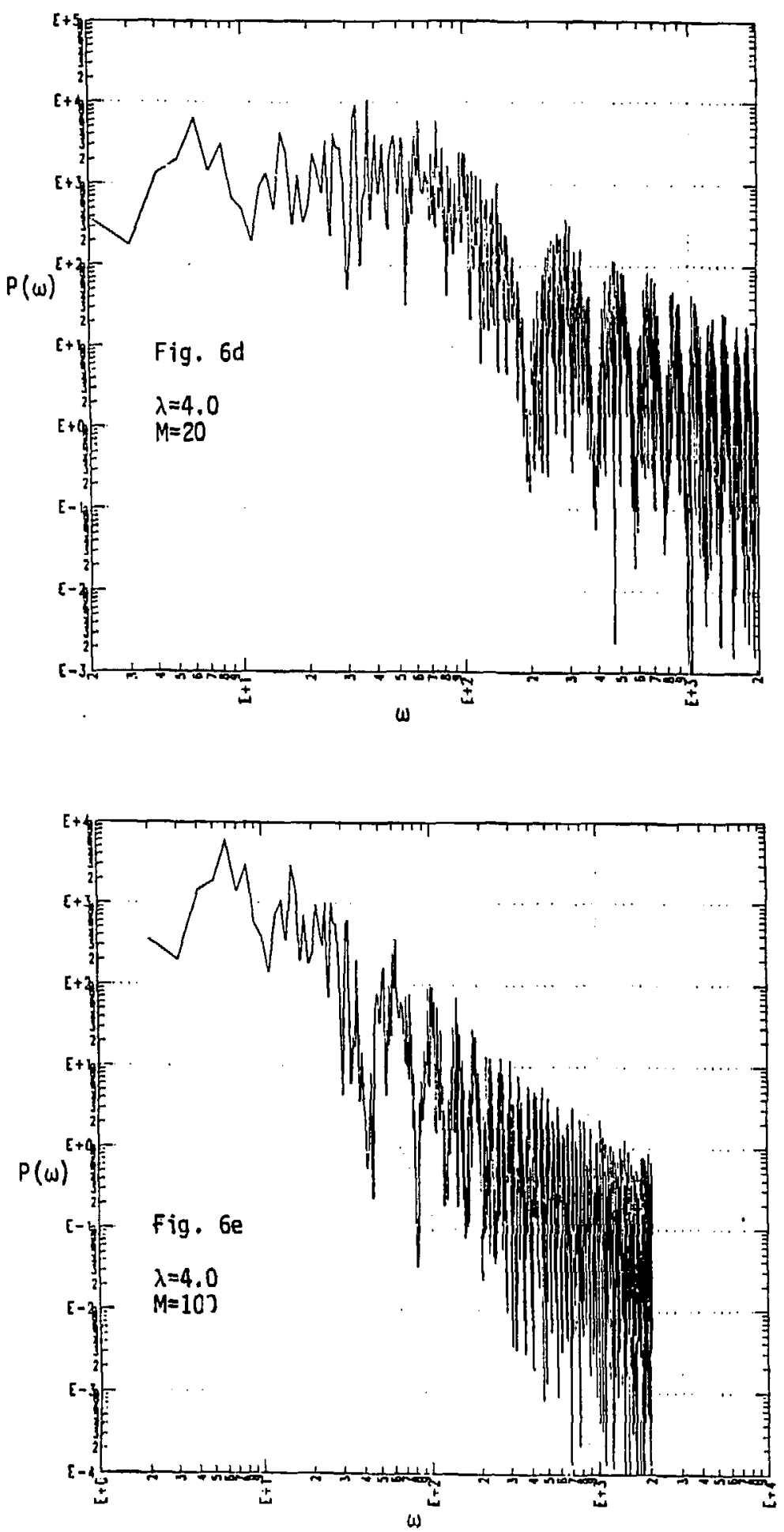
$-28-$
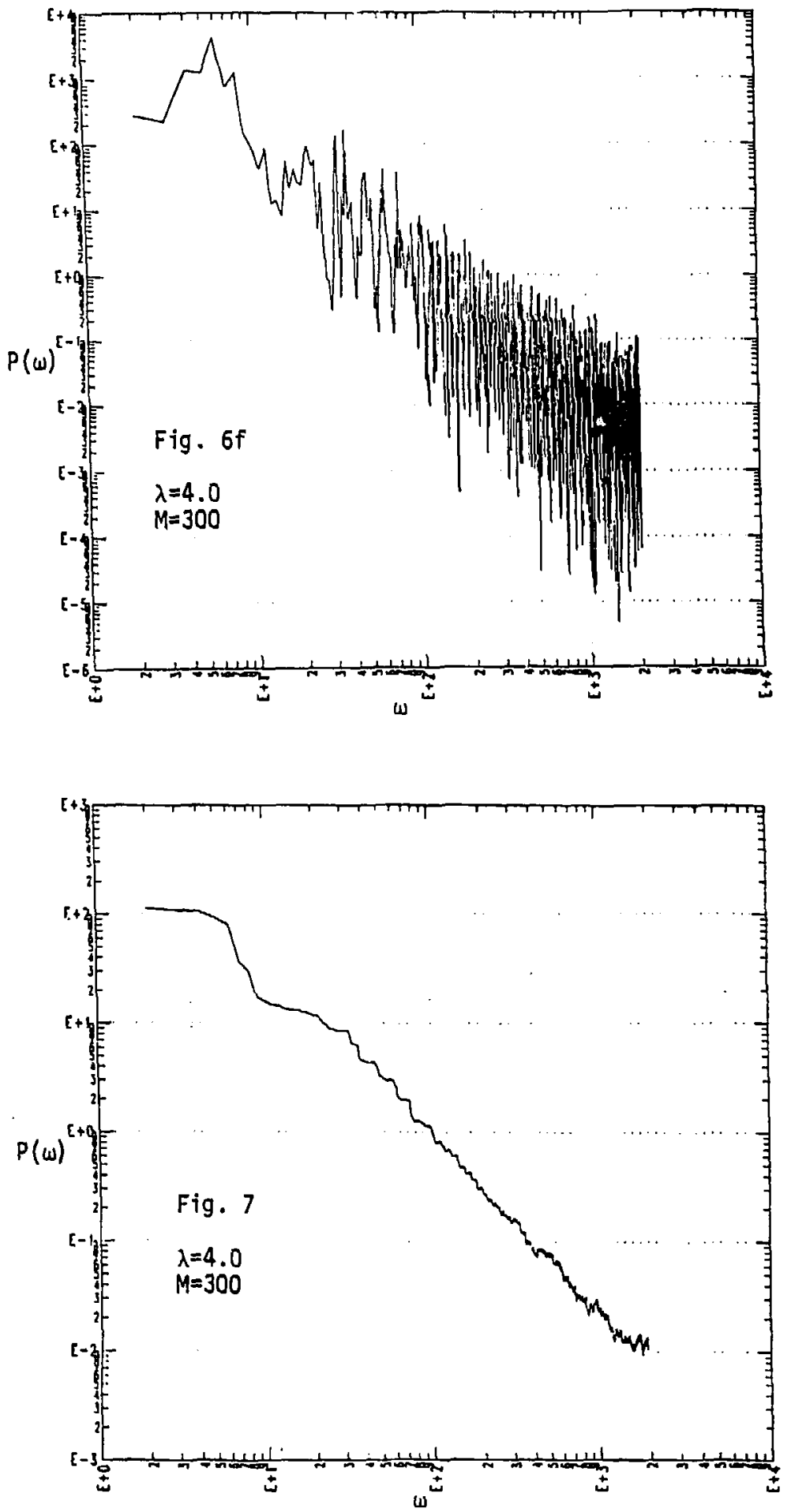

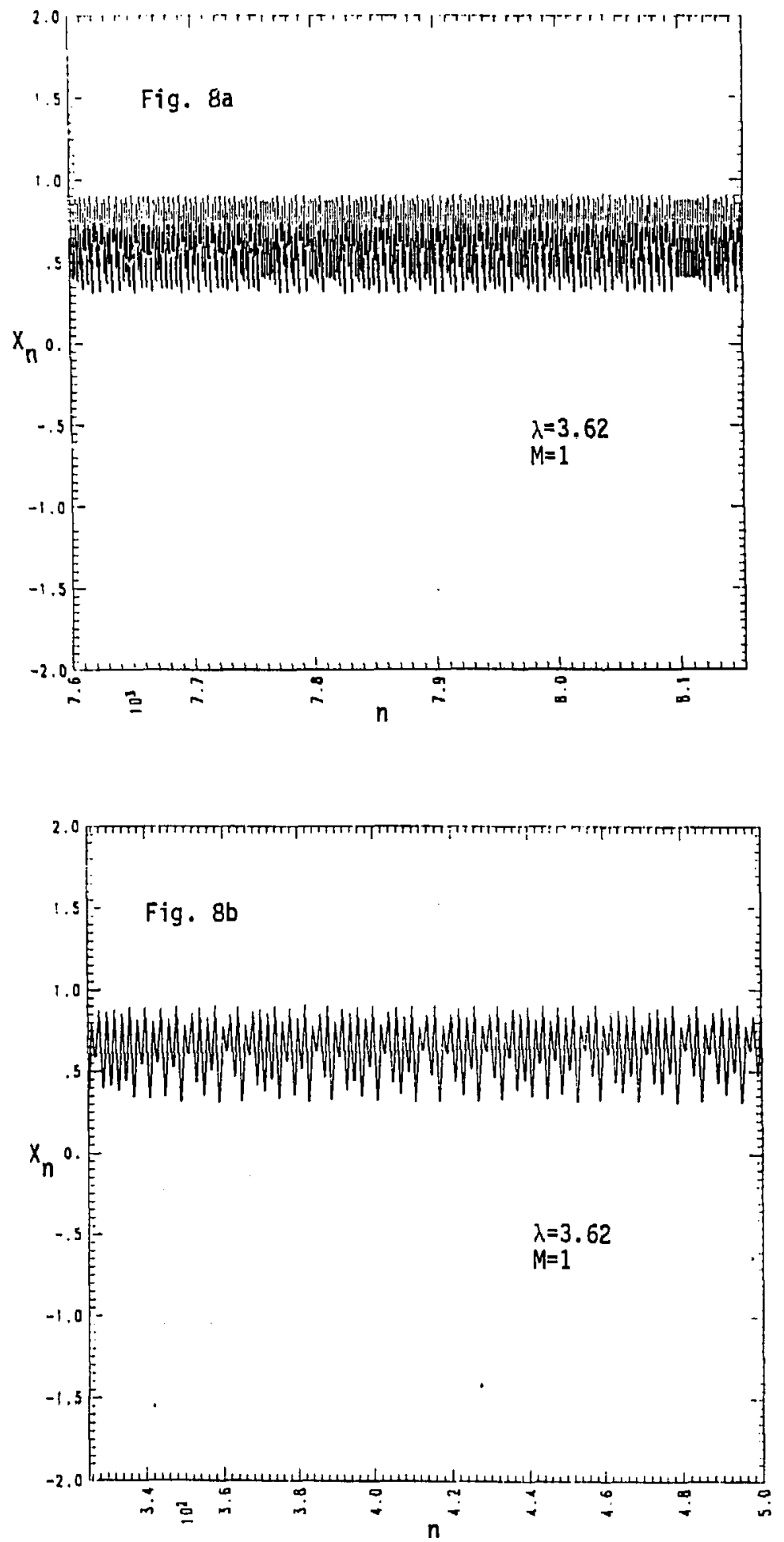
$-30-$
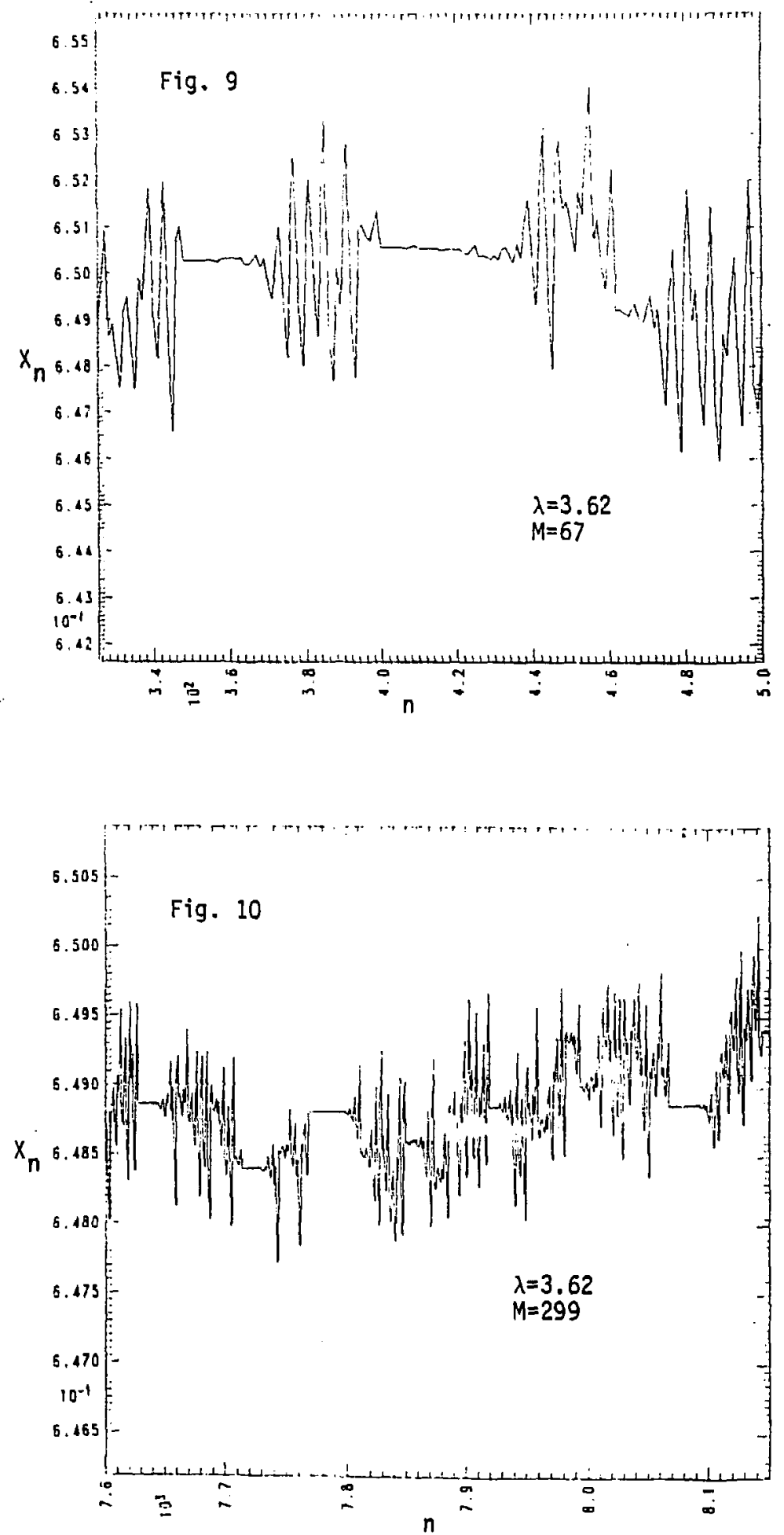

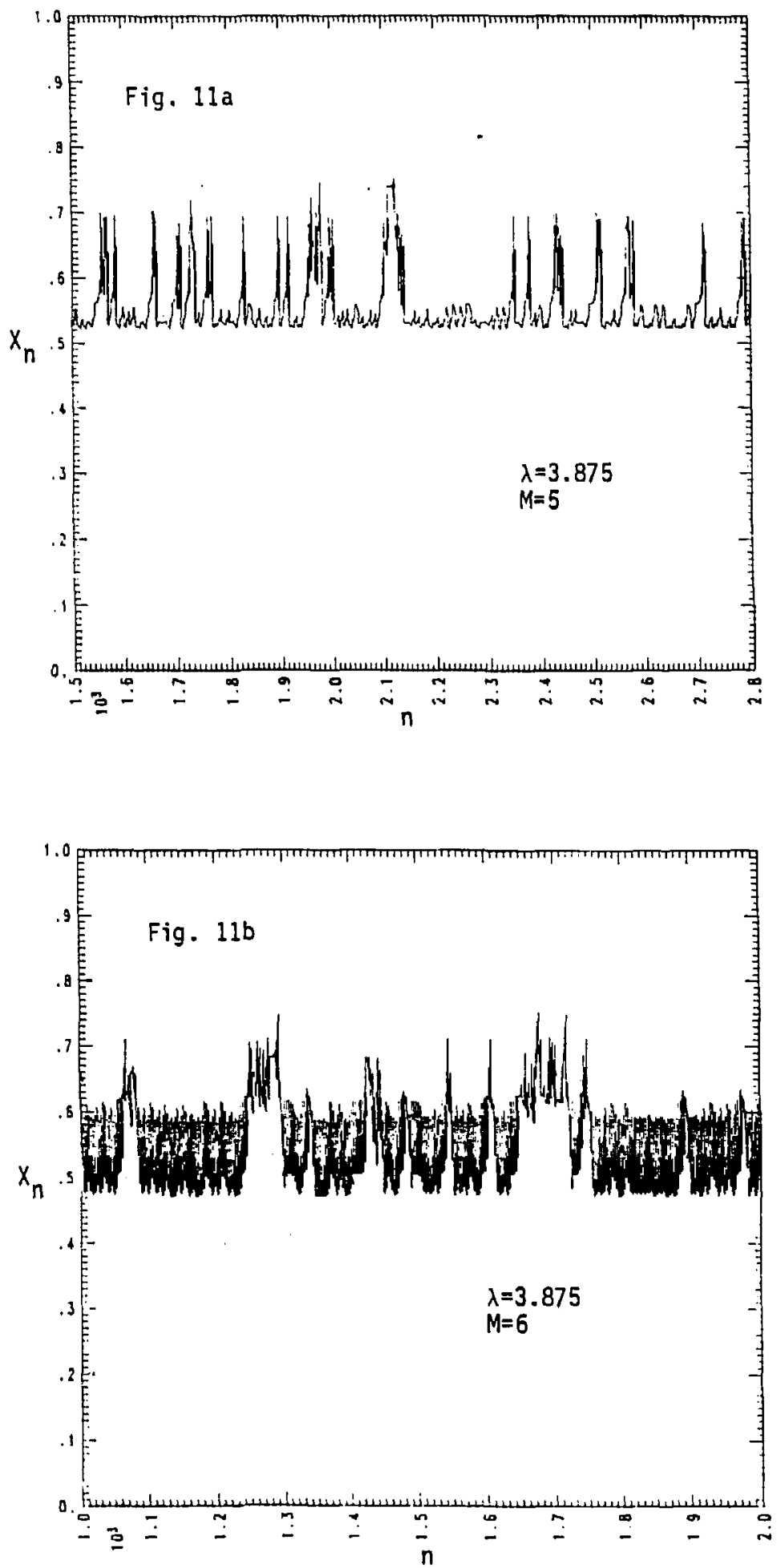
$-32-$
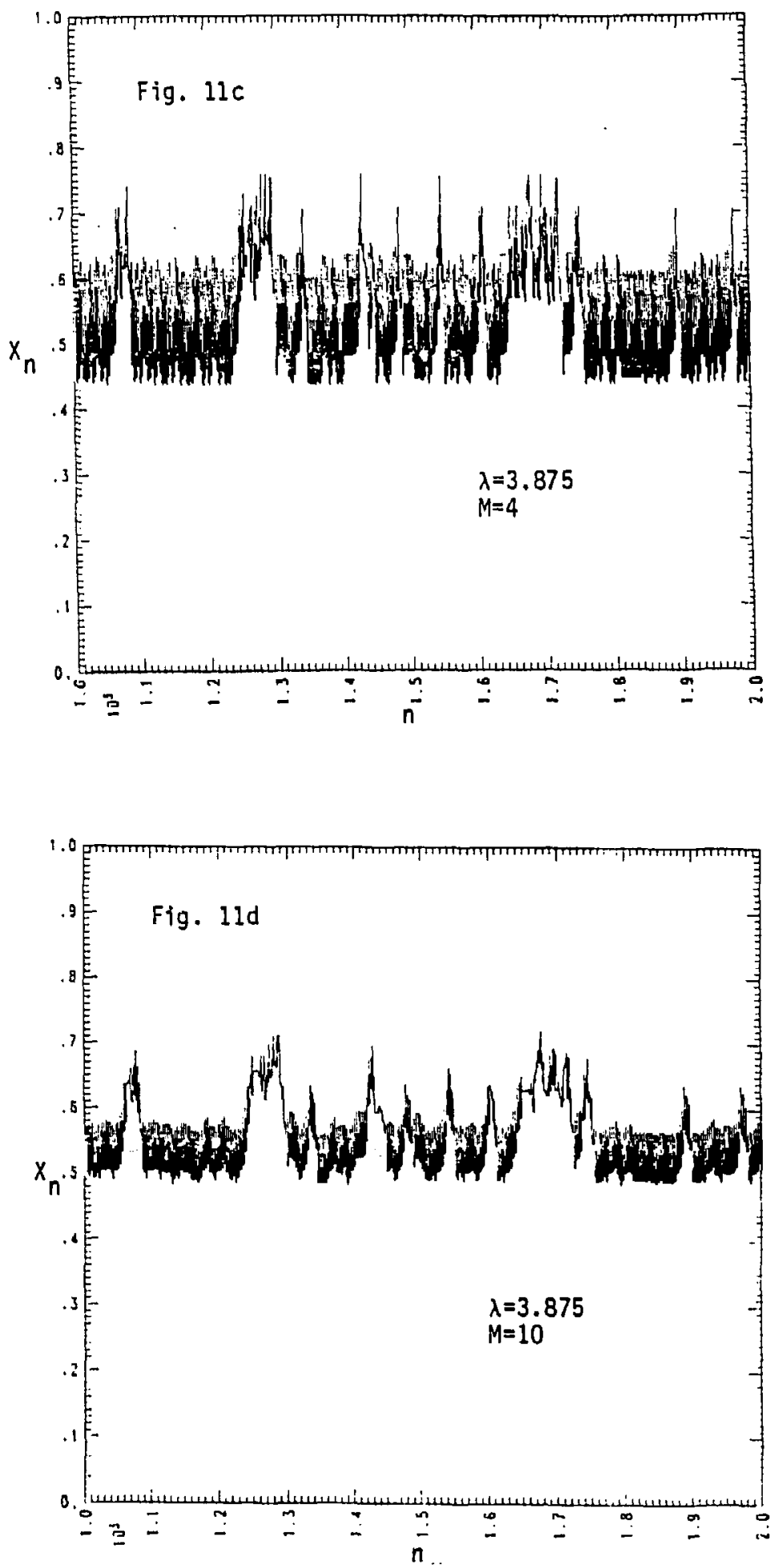


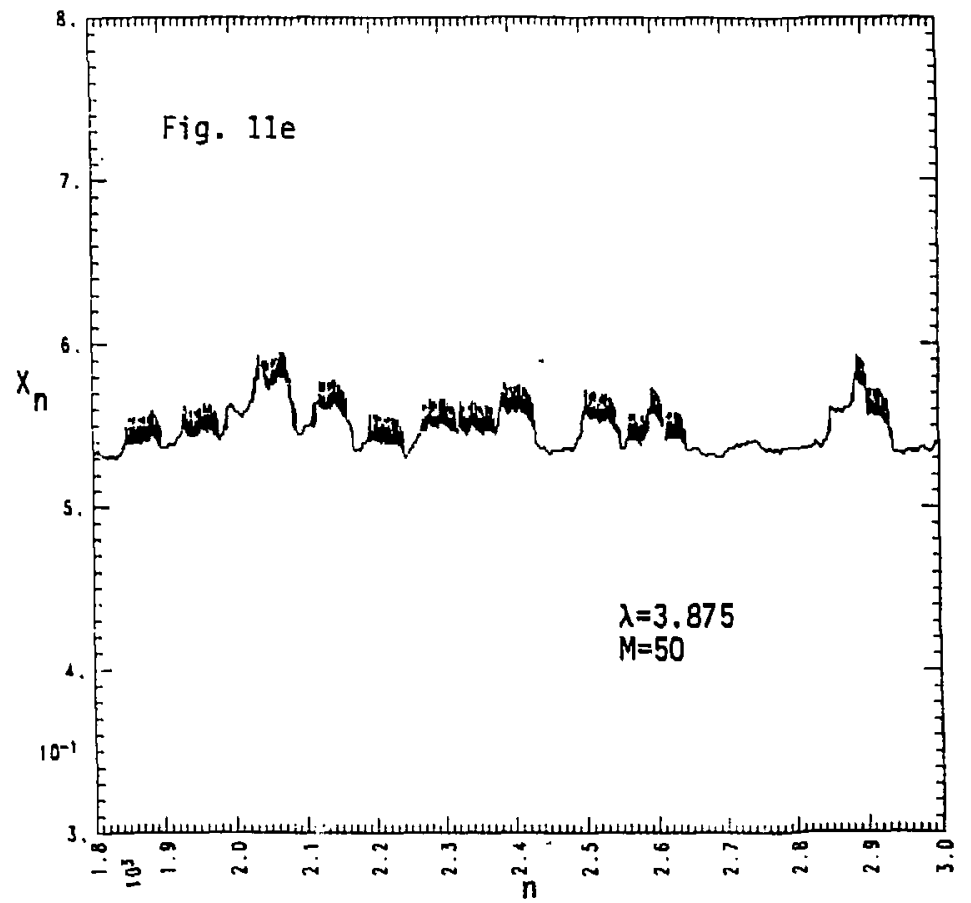


$-34-$
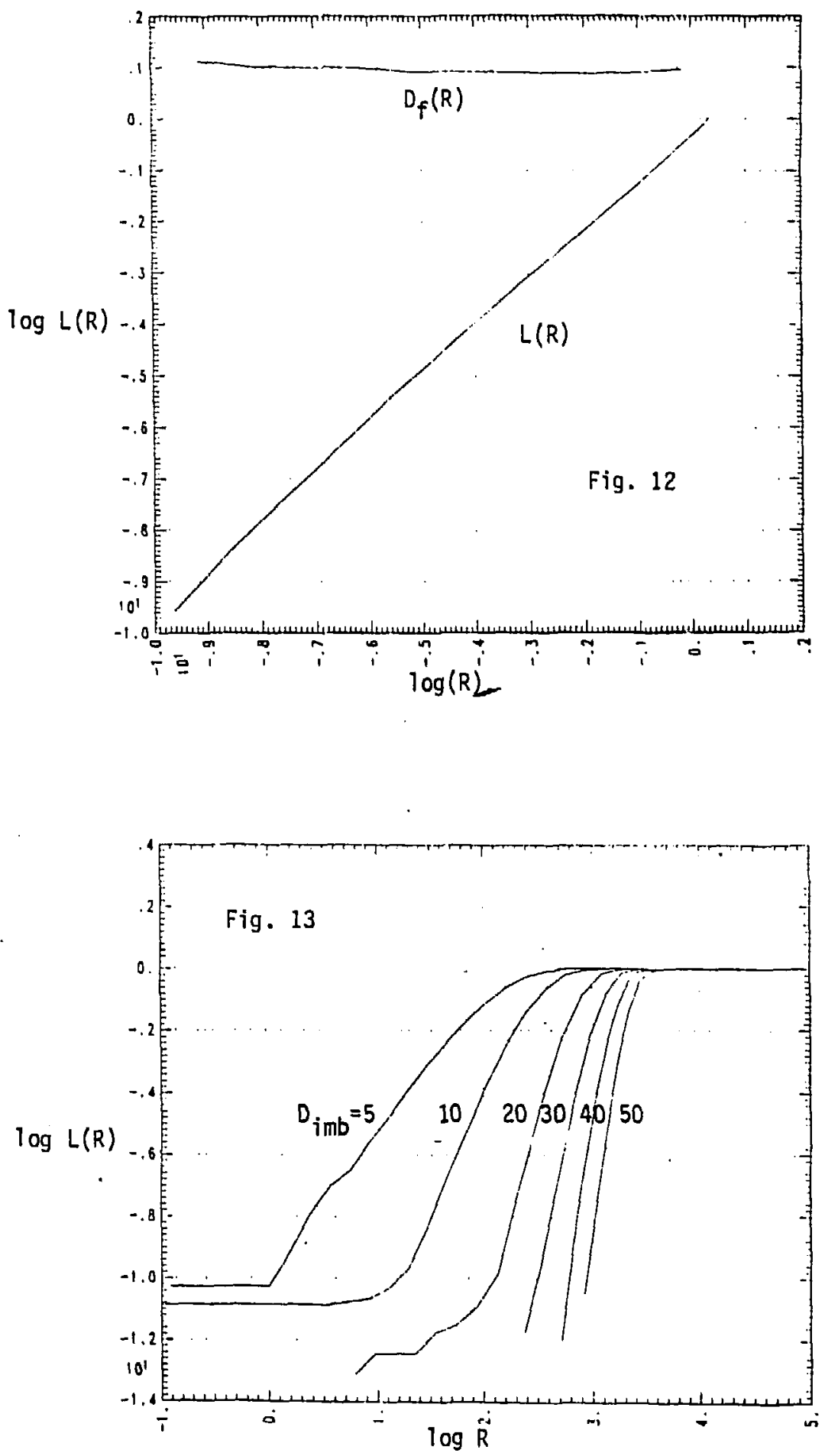